\title{
Novel Polarization Dependence in Diffusing-Wave Spectroscopy of Crystallizing Colloidal Suspensions
}

\author{
Subrata Sanyal, ${ }^{1}$ Ajay K. Sood, ${ }^{1, *, \dagger}$ S. Ramkumar,' Sriram Ramaswamy, ${ }^{2, *}$ and N. Kumar ${ }^{1, *}$ \\ ${ }^{1}$ Department of Physics, Indian Institute of Science, Bangalore 560 012, India \\ 'Centre for Theoretical Studies, Indian Institute of Science,Bangalore 560 012, India
}

\begin{abstract}
We report an unexpected behavior of the intensity autocorrelation functions $C_{V V}(t)$ (polarized) and $C_{H H}(t)$ (depolarized) of multiply scattered light from dense colloidal polyball crystals under certain conditions. We find that $C_{V V}(t)$ saturates at large times as expected for a frozen phase, while $C_{H H}(t)$ decays to zero in a short time $\boldsymbol{s}$ in a fluid. We present a new phenomenological model for this behavior based on decoupled translational and orientational fluctuations in a weakly depolarizing medium. Our study highlights the greater sensitivity of depolarized diffusing-wave spectroscopy as a probe of the dynamics of the medium.
\end{abstract}

The recently developed technique of diffusing-wave spectroscopy (DWS) [1-4] has made it possible to use light-scattering methods [5] to study the nature of dynamic correlations even in media such as concentrated colloidal suspensions $[3,4]$ and foams $[6]$, in which the light is highly multiple scattered. Most DWS studies of colloidal suspensions have focused on the liquid rather than the crystalline phase and the question of polarization dependence in particular has been looked at $[7,8]$ only in the liquid phase.

In this Letter, we report a striking and altogether unexpected polarization dependence of the DWS signal from colloidal crystals under certain conditions, and present a theoretical model which rationalizes our observations. Briefly, we find that for a nominally (poly)crystalline colloidal suspension, the time correlation of the depolarized part of the multiply scattered electric field decays to zero as in a liquid while that of the polarized part is nondecaying as expected of a solid phase. For samples aged for several weeks, however, the depolarized signal also becomes nondecaying.

In conventional quasielastic single light scattering (QELS) [5], the temporal autocorrelation $G_{1}(t)$ of the scattered electric field from a system of noninteracting, diffusing particles decays as $e^{-t / \tau_{c}}$ with $\tau_{c}=\left(D_{0} q^{2}\right)^{-1}$, where $D_{0}$ is the self-diffusion coefficient of the particles, $q=\frac{4 \pi}{\lambda_{L}} \sin (\theta / 2)$ is the scattering wave vector, $\theta$ the scattering angle, and $\lambda_{L}$ the wavelength (in the scattering medium) of the light used. In DWS, however, the averaging over photon paths and $q$ yields $[1,9]$, for the near backscattering direction, $G_{1}(t) \propto \exp \left[-\gamma\left(6 t / \tau_{0}\right)^{1 / 2}\right]$ where $\tau_{0}=\left(D_{0} k^{2}\right)^{-1}$, with $k=2 \pi / \lambda_{L}$. The parameter $\gamma$ depends on the polarization of the scattered light and on the ratio $l_{*} / l$ of the transport mean free path $l_{*}$ (the mean path length required to randomize the propagation direction) to the scattering mean free path $l$ (the mean distance between successive scattering events). For the case of interacting particles, it has been shown that [7], with some simplifying assumptions, $G_{1}(t) \propto$ $\exp \left[-\gamma\left\{6 k_{0}^{2} W(t)\right\}^{1 / 2}\right]$ where $W(t)$ is the mean square displacement of a particle in time $t$.

We note that with vertically polarized incident light while both $C_{V V}(t) \equiv\left\langle I_{V}(t) I_{V}(0)\right\rangle /\left\langle I_{V}\right\rangle^{2}-1$ and $C_{H H}(t) \equiv\left\langle I_{H}(t) I_{H}(0)\right\rangle /\left\langle I_{H}\right\rangle^{2}-1$ respond to translational motion $[7,8]$, only $C_{H H}$ is sensitive to changes in the local dielectric anisotropy and hence contains information about the correlations of orientational fluctuations as well.

We use samples of $0.115 \mu \mathrm{m}$ diameter charged polystyrene spheres (Seradyn, U.S.A.) in water, with volume fraction $\phi=0.03$, contained in a cylindrical quartz cell of $8 \mathrm{~mm}$ diameter with a mixed bed of ion-exchange resins at the bottom of the cell to reduce the ionic impurity. Light from $\mathrm{Kr}^{+}$laser $\left(\lambda_{L}=647.1 \mathrm{~nm}\right)$ is scattered at $\theta=165^{\circ}$ and the normalized intensity autocorrelation $G_{2}(t)\left[=C_{V V}(t)\right.$ or $\left.C_{H H}(t)\right]$ is measured using a Malvern correlator (model 7032CE), $G_{1}(t)$ is extracted using $G_{2}(t)=\mathrm{f}\left|G_{1}(t)\right|^{2}$, where constant $\mathrm{f}$ is determined by the system optics.

Our observations are summarized in Figs. 1 and 2. The inset of Fig. 1 shows the fluid-phase $G_{2}(t)$ before adding resins, which fitted well to the form $f \exp \left[-2 \gamma\left(6 t / \tau_{0}\right)^{1 / 2}\right]$ with $\tau_{0}=1.8086 \mathrm{msec}$ and $\gamma=1.89$ for $C_{V V}$ and 2.87 for $C_{H H}$. The value of $D_{0}$ taken for estimating $\tau_{0}$ via Stokes's law corrected for the hydrodynamic interaction $[10]$ is $D_{0}=\left(k_{B} T / 6 \pi \eta a\right)(1-1.8 \phi)$ where $\eta$ is the viscosity of water $(=0.01089 \mathrm{P})$ and $a$ the radius of the particles $(=0.0577 \mathrm{pm})$ determined from QELS experiments. That this behavior is entirely consistent with expectations and, in particular, that $f$ is very close to its ideal value of unity $\left(f_{V V}=0.99\right.$ and $\left.f_{H H}=1.06\right)$, reflects the high quality of our data and gives us confidence in our surprising new results.

The remarkable main graphs of Fig. 1 correspond to a microcrystalline suspension, confirmed by its Braggiridescence, obtained by waiting for about $120 \mathrm{~h}$ after adding resins. The measurements apply to a region $5 \mathrm{~mm}$ above the top of the resin bed. We see that while $C_{V V}$ decays to a nonzero constant, as expected for a frozen phase, $C_{H H}$ decays to zero at essentially the same rate 


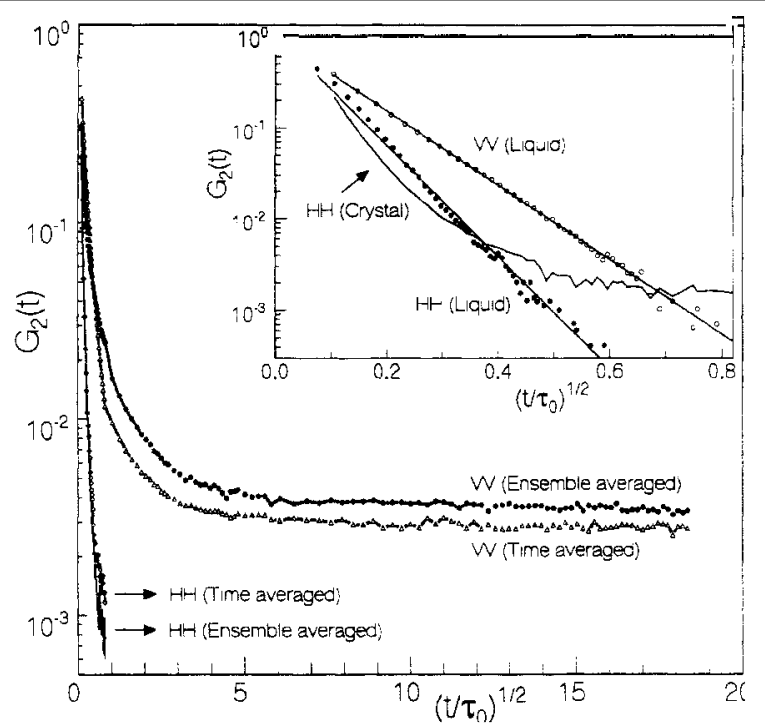

FIG. 1. The polarized (VV) and depolarized (HH) intensity autocorrelation functions $G_{2}(t)$ (while freezing) versus $\left(t / \tau_{0}\right)^{1 / 2}$ (the labels time and ensemble averaged are explained in the text). Inset shows $G_{2}(t)$ in the liquid state (circles), before adding resins, their fits (straight lines), and the decay of the depolarized "ensemble averaged" $G_{2}(t)$ in the frozen state (solid line).

as in the liquid (the inset). Transmission measurements done in the same state also show a nondecaying $C_{V V}$, implying that the sample is throughout in a state of arrested translation motion (nominally solid).

Since the system is in a frozen but disordered (i.e., nonergodic) state. we have averaged the correlation functions obtained from ten spatially separated regions in the suspension. The curves so obtained are labeled "ensemble averaged" in Fig. 1, while those from a single region are called "time averaged."

Furthermore, after about 7 weeks $C_{H H}$ also eventually acquires the time-persistent piece expected of a crystal or a glass [see Fig. 2(a)]. Lastly, if we reverse the experiment by adding ionic impurities to a well-formed colloidal crystal (with nondecaying $C_{V V}$ and $C_{H H}$ ) the resulting states for two values of impurity concentration are shown in Fig. 2. In Fig. 2(b), we see clearly the extraordinary intermediate state earlier observed in the freezing studies, and in Fig. 2(c), we find again ordinary liquidlike behavior. If we assume that the intermediate state is an imperfect crystal of some kind, clearly the $H H$ correlations are more sensitive to the type of disorder present.

We attempt to understand these results through a model which is also of interest as a new treatment of waves in random media. We work in the limit $\lambda_{L}>$ $a \phi^{-1 / 3}(>a)$, unlike the usual treatments, which are for $\lambda_{L} \ll a \phi^{-1 / 3}$. In our case, several particles are contained within a (wavelength) ${ }^{3}$, so that the local, instantaneous dielectric tensor (a) is anisotropic and (b) can be treated as a smoothly varying field. i.e.,

$$
\epsilon_{i j}(\mathbf{r}, t)=\epsilon_{0}[1+\mathcal{I}(\mathbf{r}, t)] \delta_{i j}+\epsilon_{0} \mathcal{A}_{i j}(\mathbf{r}, t)
$$

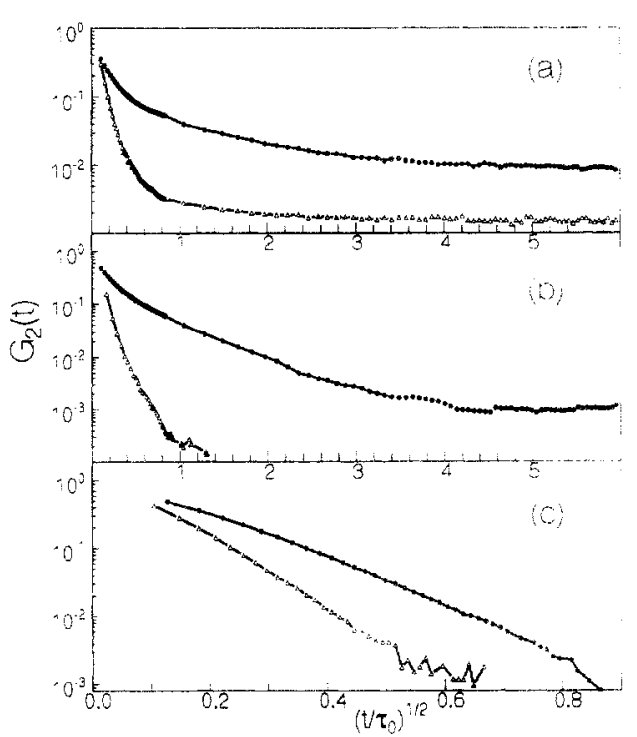

FIG. 2 Results of the melting experiment on the same sample as in Fig. 1 at $12 \mathrm{~mm}$ above the resin bed about 7 weeks after adding ion-exchange resins. (a) Both $V V$ and $\mathrm{H} \mathrm{H}$ correlations are nondecaying in the well-formed iridescent crystalline sample. (b) With an addition of 40 microequivalents $/ 1$ of $\mathrm{HCl}$ in the sample (which still remains iridescent). the unexpected simultaneous existence of a nondecaying $C_{\mathrm{VI}}$ and a liquidlike decaying $C_{H H}$, as seen in Fig. 1, is repeated (c) An addition of 400 microequivalents $/ 1$ of $\mathrm{HCl}$ melts the sample to liquid in the region probed.

where $\epsilon_{0} \mathcal{I}$ and $\epsilon_{0} \overrightarrow{\mathcal{A}}$ are the local fluctuations in the isotropic and anisotropic parts. respectively, of $\epsilon_{\imath \jmath}$, and $\epsilon_{0}$ is the average dielectric constant of the medium which is isotropic. We assume that the randomization of the direction of propagation, and hence the diffusion paths followed by the light, are determined by $\mathcal{I}$, with $\overleftrightarrow{\mathcal{A}}(\ll \mathcal{I})$ serving only to disorient the polarization. As is usual in weak-scattering treatments of DWS [11], we ignore the interference between the scattered electric fields due to distinct paths.

Along each of the random-walk paths $\mathbf{R}(s)$ (where $s$ is the path parameter) traced by the light, we assume that apart from the effect of $\overleftrightarrow{\mathcal{A}}$, the polarization vector is "Fermi transported," i.e.. rotates at a rate equal to minus the torsion [12]. We therefore use a moving [13] coordinate system along each path. with the $z$. $\mathrm{y}$, and $x$ axes being, respectively, the unit tangent $\left(\frac{\partial \mathbf{R}}{\partial s} /\left|\frac{\partial \mathbf{R}}{\partial s}\right|\right) \equiv \hat{\mathbf{t}}(s)$, unit normal $\frac{\partial^{2} \mathbf{R}}{\partial s^{2}} /\left|\frac{\partial^{2} \mathbf{R}}{\partial s^{2}}\right| \equiv \hat{\mathbf{n}}(s)$, and binormal $\hat{\mathrm{b}}(s)=\hat{\mathrm{n}}(s) \times \hat{\mathrm{t}}(s)$. We need only to keep track of the projection of $\mathcal{A}_{i j}$ into the b-n plane. The dephasing due to $\mathcal{I}(\mathbf{r}, t)$ affects both $C_{V V}$ and $C_{H H}$ in the same way as would be seen by MacKintosh and John's treatment [7], and cannot therefore explain our observations.

We begin with the wave equation for $\mathbf{E}_{\omega}(\mathbf{r}, t)$, the wth Fourier component of the electric field, with the usual assumption that the time dependence in $E_{\omega}(\mathrm{r}, t)$ is purely because the medium is changing in time: 


$$
-\left(\nabla^{2}+k_{0}^{2}\right) \mathbf{E}_{\omega}=k_{0}^{2}[\stackrel{\leftrightarrow}{\mathcal{A}}(\mathbf{r}, t)+\mathcal{I}(\mathbf{r}, t)] \mathbf{E},
$$

where $k_{0}=\omega \sqrt{\epsilon_{0}} / c ; c$ is the speed of light $a n$ vacuo. Suitable statistical properties for $\mathcal{I}$ and $\overleftrightarrow{\mathcal{A}}$ will be introduced later. Along a path $\mathbf{R}(s)$, the electric field then evolves with $s$, according to

$$
\begin{aligned}
-\frac{\partial^{2}}{\partial s^{2}}\left(\begin{array}{c}
E_{\|}(s) \\
E_{\perp}(s)
\end{array}\right)= & k_{0}^{2} \stackrel{\leftrightarrow}{\mathcal{A}}(\mathbf{R}(s), t)\left(\begin{array}{c}
E_{\|}(s) \\
E_{\perp}(s)
\end{array}\right) \\
& +k_{0}^{2}[\mathcal{I}(\mathbf{R}(s), t)+1]\left(\begin{array}{c}
E_{\|}(s) \\
E_{\perp}(s)
\end{array}\right)
\end{aligned}
$$

where $\|$ and $\perp$ are directions along and perpendicular to the parallel-transported polarization, respectively, and we have suppressed the subscript $w$. Since we are concerned only with the part of $\overleftrightarrow{\mathcal{A}}$ that acts in the b-n plane, we can model it by a random symmetric traceless tensor in two dimensions:

$$
\mathcal{A}_{i j}=a_{0}\left(N_{i} N_{j}-\frac{1}{2} \delta_{i j}\right)
$$

where $N_{i}=N_{i}(\mathrm{R}(s), t)$ is the local, instantaneous, axis of anisotropy of the projection into the $\mathbf{b}-\mathbf{n}$ plane of the dielectric tensor and $a_{0} \equiv a_{0}(\mathbf{R}(s), t)$ is the overall amplitude of the anisotropic local fluctuations.

For $a_{0}=0$, to lowest order in $\mathcal{I}(s), \mathrm{E}(s)=$ $\mathrm{e}^{2 \int^{s} k\left(s^{\prime}\right) d s^{\prime}} \mathrm{E}(0)$, where $k(s)=k_{0} \sqrt{1+\mathcal{I}(s)}$. For $a_{0} \neq 0$, it is still useful to separate the effects of $\mathcal{I}$ from those of $\overleftrightarrow{\mathcal{A}}$ by defining

$$
\mathcal{E}(s)=e^{-i \int^{s} k\left(s^{\prime}\right) d s^{\prime}} \mathbf{E}(s),
$$

where the $s$ evolution of $\mathcal{E}$ is governed purely by $\overleftrightarrow{\mathcal{A}}$. Equation (3) then yields

$$
\begin{aligned}
& i \frac{\partial}{\partial s}\left(\begin{array}{l}
\mathcal{E}_{\|}(s) \\
\mathcal{E}_{\perp}(s)
\end{array}\right) \\
& \quad=-\frac{k_{0}}{4} a_{0}(s)\left(\begin{array}{cc}
\cos 2 \theta(s) & \sin 2 \theta(s) \\
\sin 2 \theta(s) & -\cos 2 \theta(s)
\end{array}\right)\left(\begin{array}{c}
\mathcal{E}_{\|}(s) \\
\mathcal{E}_{\perp}(s)
\end{array}\right)
\end{aligned}
$$

where terms of order $\frac{\partial^{2}}{\partial s^{2}}$ have been dropped in a "forward propagating wave" approximation [14], valid for $a_{0}$ and $\theta$ varying slowly on the scale of $\lambda_{L}$. Here $\theta$ is the angle between $\hat{\mathrm{N}}$ and $\mathbf{n}$.

Ignoring for the moment the actual tame dependence of $\theta$ and $a_{0}$, we see that for each path $\mathbf{R}(s)$, Eq. (6) is the Schrodinger equation for a quantum spin- $\frac{1}{2}$ state with Hamiltonian $31=-\frac{1}{2} \sigma \cdot h(s)$ in a magnetic field $\mathrm{h}(s)=k_{0} a_{0}(s)(\sin 2 \theta(s), 0, \cos 2 \theta(s))$, lying in the $x-z$ plane and fluctuating with respect to the "time" s.

Let us work in the "interaction picture," treating the $\sigma_{x}$ term as the interaction. This choice is motivated by the fact that the initial state is an eigenstate of $\sigma_{z}$. We define

$$
\begin{aligned}
& \beta(s)=\frac{k_{0}}{2} \int^{s} a_{0}\left(s^{\prime}\right) \sin 2 \theta\left(s^{\prime}\right) d s^{\prime}, \\
& \gamma(s)=\frac{k_{0}}{2} \int^{s} a_{0}\left(s^{\prime}\right) \cos 2 \theta\left(s^{\prime}\right) d s^{\prime}
\end{aligned}
$$

and let

$$
\left(\begin{array}{c}
\mathcal{E}_{\|} \\
\mathcal{E}_{\perp}
\end{array}\right)=e^{i \gamma(s) \sigma_{z}}\left(\begin{array}{c}
\tilde{\mathcal{E}_{\|}} \\
\tilde{\mathcal{E}}_{\perp}
\end{array}\right)
$$

Then

$$
\begin{aligned}
i \frac{\partial}{\partial s}\left(\begin{array}{c}
\tilde{\mathcal{E}}_{\|} \\
\tilde{\mathcal{E}}_{\perp}
\end{array}\right)= & -\frac{k_{0} a_{0}(s)}{2} \sin 2 \theta(s) \\
& \times\left(e^{-i \gamma(s) \sigma_{z}} \sigma_{x} e^{i \gamma(s) \sigma_{\boldsymbol{z}}}\right)\left(\begin{array}{c}
\tilde{\mathcal{E}}_{\|} \\
\tilde{\mathcal{E}}_{\perp}
\end{array}\right) .
\end{aligned}
$$

Of course, we cannot solve Eq. (9) exactly either since $\mathcal{H}$ does not commute with itself at different "times" s, so that the solution involves a "time-ordered" exponential. We, however, solve the problem only in the approximation where the consequent interference between the spinflip and non-spin-flip terms is neglected. This is reasonable since the coefficients of $\sigma_{x}$ and $\sigma_{z}$ involve $\sin 2 \theta(s)$ and $\cos 2 \theta(s)$, respectively, and these are expected to be statistically orthogonal. Then, Eq. (9) becomes

$$
i \frac{\partial}{\partial s}\left(\begin{array}{l}
\tilde{\mathcal{E}_{\|}} \\
\mathcal{E}_{\perp}
\end{array}\right)=-\frac{k_{0}}{2} a_{0}(s) \sin 2 \theta(s) \sigma_{x}\left(\begin{array}{c}
\tilde{\mathcal{E}}_{\|} \\
\mathcal{E}_{\perp}
\end{array}\right) \text {. }
$$

Solving Eq. (10) (which is trivial) we see that

$$
\left(\begin{array}{c}
\tilde{\mathcal{E}}_{\jmath}(s, t) \\
\tilde{\mathcal{E}}_{\perp}(s, t)
\end{array}\right)=\left[\cos \beta(s, t)+i \sin \beta(s, t) \sigma_{x}\right]\left(\begin{array}{c}
\tilde{\mathcal{E}}_{j}(0, t) \\
\mathcal{E}_{\perp}(0, t)
\end{array}\right)
$$

We approximate $a_{0} \sin 2 \theta$ and $a_{0} \cos 2 \theta$ by mutually uncorrelated Gaussian random noise sources, with zero mean, and with covariances

$\frac{\mathrm{k}_{2}^{2}}{2}\left\langle a_{0}(s, t) \sin 2 \theta(s, t) a_{0}(0,0) \sin 2 \theta(0,0)\right\rangle$

$$
=S \delta(s) e^{-t / \tau_{A}},
$$

$\frac{k_{0}^{2}}{2}\left\langle a_{0}(s, t) \cos 2 \theta(s, t) a_{0}(0,0) \cos 2 \theta(0,0)\right\rangle$

$$
=C \delta(s) e^{-t / \tau_{I}},
$$

$\frac{k_{0}^{2}}{\hbar}\left\langle a_{0}(s, t) \cos 2 \theta(s, t) a_{0}(0,0) \sin 2 \theta(0,0)\right\rangle=0$.

This amounts to assuming a local optical axis uncorrelated in space and weakly correlated in time, which is reasonable for a system without significant quadrupolar orientational correlations.

In the reference frame in which Eq. (3) is written, the initial condition is $\tilde{\mathcal{E}}_{\|}(0, t)=E_{0}$ and $\tilde{\mathcal{E}}_{\perp}(0, t)=0$. We can then solve for $\left(\begin{array}{l}\tilde{\varepsilon}_{\mid(s, t)} \\ \tilde{\varepsilon}_{\perp}(s, t)\end{array}\right)$ and transform back using Eq. (8) to get $\left(\begin{array}{l}\mathcal{E}_{\|}(s, t) \\ \varepsilon_{\perp}(s, t)\end{array}\right)$. Using Eq. (14) to decouple the correla- 
tions of $\gamma$ and $\beta$, we find that the correlation functions of the electric field components along and perpendicular to the Fermi-transported initial polarization are. respectively,

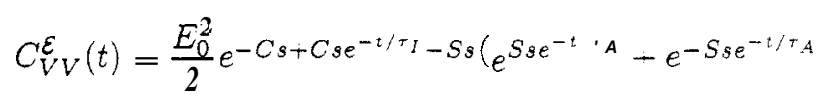

and

$C_{H H}^{\mathcal{E}}(t)=\frac{E_{0}^{2}}{2} e^{-C s+C s e^{-t / \tau}-S s}\left(e^{S s e^{-t / \tau_{A}}}-e^{-S s e^{-t / \tau} A}\right)$.

For $\mathrm{t} \gg \tau_{I}, \tau_{A}$, we see that

$$
C_{V V}^{\mathcal{E}}(t) \longrightarrow E_{0}^{2} e^{-(S+C)_{s}}
$$

while

$$
C_{H H}^{\mathcal{E}}(t) \longrightarrow E_{0}^{2} S s e^{-(S+C) s} e^{-t / \tau_{A}} .
$$

The measured correlation functions are. of course. $C_{V V}=\left|C_{V V}^{\mathcal{E}}\right|^{2}$ and $C_{H H}=\left|C_{H H}^{\mathcal{E}}\right|^{2}$. To claim that the observed lab frame $C_{V V}$ and $C_{H H}$ correspond to those we have calculated requires that in the absence of anisotropic fluctuations, the output and incident polarizations are nearly parallel. This would be exact only if the multiple scattering were confined to a plane perpendicular to the incident polarization. Inasmuch as the observed output scattered intensities are in the ratio $l_{Y V H}^{l_{V V}} \simeq \mathbf{3}$, our approximation is not too bad. With this preamble, we return to Eqs. (15)-(18), whence we see clearly that the effect of decorrelated onentatzonal fluctuations is to cause $C_{H H}$ to decay, while leaving $C_{V V}$ unaffected. More precisely, the ratio $C_{H H} / C_{V V}$ decays as $e^{-t / \tau_{A}}$ for $\tau \gg \tau_{A}$, independent of $t / \tau_{I}$. Thus, if other dynamic fluctuations of a purely translational type are frozen, then weak orientational fluctuations can decorrelate $C_{H H}$ in preference to $C_{V V}$. Of course, we must average over a suitable distribution of path lengths $P(s)$, but the result that $C_{H H}$ decays while $C_{V V}$ does not is unchanged.

It is appropriate to remark at this point on the relation between our approach and that of MacKintosh and John [7]. Note that for short times $t<\tau_{A}, \tau_{I}$, the overall time dependence of the correlation functions is of the form $e^{- \text {(const) } s t / \tau_{I}}$. This. when averaged using $P(s) \propto \exp \left[-\right.$ (const) $\left.\frac{l^{2}}{s}\right]$, for backscattering. will give decays which are exponential in $\sqrt{t}$. Such a restriction to "short" times is implicit in [7] [see their Eqs. (3.11)(3.12)]

Our conjecture for the underlying origin of these fluctuations is as follows. Our system, in the imperfectly crystallized regime contains some anisotropic entities, perhaps interfaces between fcc- or bcc-symmetric (and hence optically isotropic) crystallites for which quadrupolar or higher shape fluctuations cost little energy and therefore contribute appreciably mainly to depolarized scattering and to the decay of $H H$ correlations. If these defects were absent, all depolarization would contribute identically to $C_{V V}$ and $C_{H H}$, and both would saturate, but in their presence, the mechanism outlined above can act to give the startling difference between $C_{V V}$ and $C_{H H}$. As time goes on, because of either the growth of the crystallites, or the annealing and "hardening" of the interfacial regions (or both), this anomalous scattering mechanism freezes out. This leaves only the translational motions of the colloidal particles, which dephase $C_{V V}$ and $C_{H H}$ in essentially the same way. A small-angle x-ray or neutron scattering study could tell us more about the state of order of the colloidal suspension in the various stages of crystallization, and allow us to refine our model of the medium. Pending such a study, we must content ourselves with pointing out this unusual and perhaps universal transient property of light multiply scattered from crystallizing colloidal suspensions.

A.K.S. and S.R. thank the Indo-French Centre for the Promotion of Advanced Research for financial support. We also thank Rajaram Nityananda for very useful discussions and a critical reading of the manuscript.

* Also at Jawaharlal Nehru Centre for Advanced Scientific Research, IISc Campus, Bangalore 560 012, India

1 Author to whom correspondence should be addressed. Electronic address: asood@physics.ernet.in

1 G. Maret and P. E. Wolf, Z. Phys. B 65, 409 (1987).

[2] I. Reund, M. Kaveh, and M. Rosenbluh, Phys. Rev. Lett. 60. 1130 (1988).

[3] D. J. Pine, D. A. Weitz, P. M. Chaikin, arid E. Herbolzheimer, Phys. Rev. Lett. 60, 1134 (1988).

[4] D. J. Pine, D. A. Weitz, G. Maret, P. E. Wolf, E. Herbolzheimer, and P. M. Chaikin, Scattering and Localization of Classical Waves in Random Media, edited by $\mathrm{P}$. Sheng (World Scientific, Singapore, 1989).

[5] B. J. Berne and R. Pecora, Dynamic Light Scattering (John Wiley, New York, 1976).

[6] D. J. Durian, D. A. Weitz. and D. J. Pine. Science 252. 686 (1991).

[7] F. C. Mackintosh and S. John. Phys. Rev. B 40. 2383 (1989).

[8] F. C. MacKintosh, J, X. Zhu, D. J. Pine, and D. A. Weitz. Phys. Rev. B 40, 9342 (1989).

[9] A. A. Golubentsev, Zh. Eksp. Teor. Fiz. 86, 47 (1984) [Sov. Phys. JETP 59, 26 (1984);

[10] G. K. Batchelor, J. Fluid Mech. 74, 1 (1976); 131, 155 (1983).

[11] M. J. Stephen, Phys. Rev. B 37, 1 (1988).

[12] L. D. Landau and E. 51. Lifshitz, Electrodynamics of Continuous Media (Pergamon, Oxford, 1963), p. 292.

[13] See, for example, B. O'Neill, Elementary Differential Geometry (Academic Press, New York, 1972).

[14] D. Gloge and D. Marcus, J. Opt. Soc. Am. 59, 1629 (1969). 